\title{
Measuring Programming Experience
}

\author{
Janet Feigenspan, \\ University of Magdeburg \\ Christian Kästner, \\ Philipps University Marburg
}

\author{
Jörg Liebig and Sven Apel, \\ University of Passau
}

\author{
Stefan Hanenberg, \\ University of Duisburg-Essen
}

\begin{abstract}
Programming experience is an important confounding parameter in controlled experiments regarding program comprehension. In literature, ways to measure or control programming experience vary. Often, researchers neglect it or do not specify how they controlled it. We set out to find a well-defined understanding of programming experience and a way to measure it. From published comprehension experiments, we extracted questions that assess programming experience. In a controlled experiment, we compare the answers of 128 students to these questions with their performance in solving program-comprehension tasks. We found that self estimation seems to be a reliable way to measure programming experience. Furthermore, we applied exploratory factor analysis to extract a model of programming experience. With our analysis, we initiate a path toward measuring programming experience with a valid and reliable tool, so that we can control its influence on program comprehension.
\end{abstract}

\section{INTRODUCTION}

In software-engineering experiments, program comprehension is frequently evaluated, for example, for the evaluation of programming-language constructs or software-development tools [3], [7], [13], [16], [26]. Program comprehension is an internal cognitive process that we cannot observe directly. Instead, controlled experiments are often conducted, in which we observe the behavior of subjects and draw conclusions about their program comprehension.

To conduct controlled experiments, we have to control confounding parameters, which influence the outcome of an experiment in addition to the evaluated concept [15]. One important confounding parameter is programming experience: The more experienced a participant, the better she understands a program compared to an inexperienced participant. (Accidentally) assigning experienced subjects to one treatment can seriously bias the results. Hence, programming experience should always be considered in such kind of experiments.

However, there is no agreed way to measure programming experience. Instead, researchers use different measures, do not specify how they measured programming experience, or do not measure it at all. However, a common understanding of programming experience can increase the validity of experiments and helps interpreting results.

Our goal is to evaluate how reliable different ways of measuring programming experience are. To this end, we conducted a controlled experiment, in which subjects completed a questionnaire that contained several typical questions related to programming experience based on a literature review. Additionally, subjects solved simple programming tasks. Then, we compared the performance in the programming tasks with the answers in the questionnaire.
As result, we identified two questions as indicator for programming experience using stepwise regression: Self estimated programming experience compared to class mates and self estimated experience with logical programming. Furthermore, we present a five-factor model that describes programming experience using exploratory factor analysis. The contributions of this paper are the following:

- Literature review about the state of the art of measuring and controlling the influence of programming experience.

- A questionnaire that contains the common questions to measure programming experience.

- Reusable experimental design to evaluate the questionnaire.

- Initial evaluation of this questionnaire with sophomores.

- Proposal toward two relevant questions and a five-factor model of programming experience.

\section{Literature REVIEW}

To get an overview of whether and how other researchers measure programming experience, we conducted a literature review. We based our review on the guidelines for systematic literature reviews provided by Kitchenham and Chartes [20]. Due to space restrictions, we only give a short summary of the extraction process and the results. We considered the years 2001 to 2010 of highly ranked conferences and journals in the domain of (empirical) software engineering and program comprehension: International Conference on Software Engineering (ICSE), International Workshop/Conference on Program Comprehension (ICPC), ${ }^{1}$ International Symposium on Software Engineering and Measurement (ESEM), ${ }^{2}$ Empirical Software Engineering Journal (ESE), Transactions on Software Engineering (TSE), Transactions on Software Engineering and Methodology (TOSEM), and European Software Engineering Conference/Foundations on Software Engineering (FSE).

To extract the papers, we started by reading title and abstract of each paper. If an experiment with human subjects was mentioned, we included the paper in our selection. If the abstract was not conclusive, we skimmed the paper and searched for the keywords (programming) experience, expert, expertise, professional, subject, and participant, which are typical for program-comprehension experiments. We extracted 288 (of 2161) papers. We read each paper of our selection and excluded those papers that evaluated a concept too far

\footnotetext{
${ }^{1}$ ICPC was a workshop until 2005 (IWPC), which we also inlcuded.

${ }^{2}$ ESEM first took place in 2007.
} 


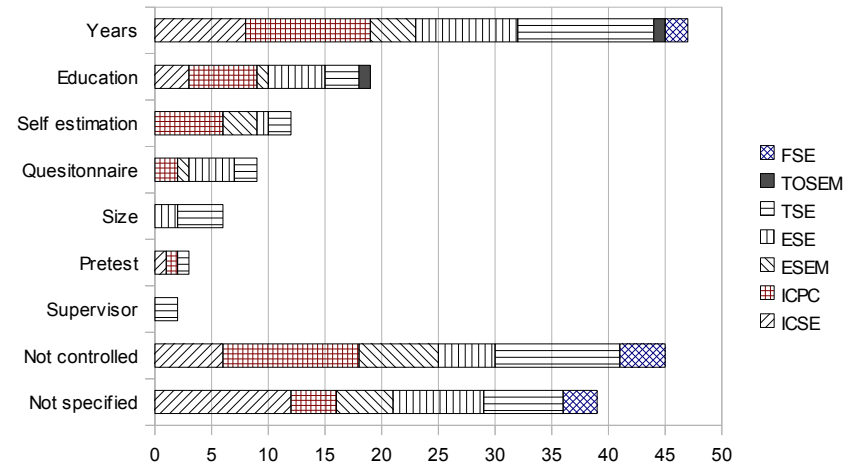

Fig. 1. Overview of how programming experience is operationalized.

away from program comprehension (e.g., cost estimation of software projects). When uncertain whether a concept was too far away (e.g., some experiments on software inspection are relevant for us depending on the design of the experiment), we discussed it until we reached an agreement. ${ }^{3}$ When still in doubt, we included the paper to have an exhaustive overview of the understanding of programming experience. After this exclusion process, we have 161 Papers in our final selection. An overview of our initial selection of papers as well as excluded and included papers is available at the project's website. ${ }^{4}$

In the selected papers, we found several ways of how programming experience was handled, which we divide into 9 categories, shown in Fig. 1. Note that the categories are not disjoint; sometimes, authors used a combination of indicators. In that case, the according paper is counted for each category.

1) Years: In most papers (47), the years a subject was programming at all or programming in a company or certain language was used to measure programming experience. For example, Sillito and others assessed the number of years a subject was programming professionally [26].

2) Education: The education of subjects was used to indicate their experience in 19 of the reviewed papers. Education includes information such as the level of education (e.g., undergraduate or graduate student) or the grades of courses. For example, Ricca and others recruited undergraduate students as low experience and graduates as high-experience subjects [25].

3) Self estimation: In twelve experiments, subjects were asked to estimate their experience themselves. For example, Bunse let his subjects estimate their experience on a five-point scale [7].

4) Questionnaire: Some authors applied a questionnaire to assess programming experience. For example, Erdogmus and others let subjects fill out a questionnaire before the experiment [11]. However, it was not specified what the questionnaire looked like.

5) Size: The size of programs subjects had written was

\footnotetext{
${ }^{3}$ The literature-review team consisted of the first author and a research assistant.

${ }^{4}$ http://fosd.net/PE
}

used as indicator in six papers. For example, Müller [23] asked how many lines of code the largest program has that subjects have implemented.

6) Pretest: In three experiments, a pretest was conducted to assess the subjects' programming experience. For example, Biffl and Grossmann [5] used a pretest to create three groups of skill levels (excellent, medium, little). However, it was not specified in the papers what the pretest looked like.

7) Supervisor: In two experiments, in which professional programmers were recruited as subjects, the supervising manager estimated the experience of subjects [3], [17].

8) Not specified/not controlled: Often, the authors state that they measured programming experience, but did not specify how. This was the case in 39 papers. Even more often (45 papers), programming experience was not mentioned at all, which threaten the validity of the corresponding papers.

To summarize, the measurement of programming experience is diverse. This could threaten the validity of the experiments, because researchers use their own definition of programming experience without validating that definition. Furthermore, conducting meta analysis on these experiments is difficult, because the influence of programming experience is not clearly defined, making the results across different experiments not comparable. To evaluate the measurement of programming experience, we created a questionnaire based on the results of the literature review.

\section{QUestionnAire}

Most measurements of programming experience we found in literature can be performed as part of a questionnaire. Only pretest and supervisor estimation require additional effort, but are also rarely used. Hence, we excluded both categories. Furthermore, we excluded the category questionnaire, because the contents of questionnaire were not specified.

We designed a single questionnaire, which includes questions of the following categories: years, education, self estimation, and size. For each category, we selected multiple questions we found in literature. Additionally, we added questions that we found in previous experiments to be related to programming experience. This way, we aim at having a more exhaustive set of indicators for programming experience and, consequently, a better definition of programming experience. Some questions are specific to students; when working with different subjects (e.g., experts), they need to be adapted.

Our goal is to evaluate which questions from which categories have the highest prediction power for programming experience. In the long run, we plan to evolve our questionnaire (by removing questions with little prediction power, and potentially adding others) into a standard questionnaire.

In Table I, we summarize our questionnaire. We also show the scale of the answers, that is how subjects should answer the questions. In column Abbreviation, we show the abbreviation of each question, which we use in the remainder. The version 


\begin{tabular}{|c|c|c|c|}
\hline Source & Question & Scale & Abbreviation \\
\hline \multirow[t]{6}{*}{$\begin{array}{l}\text { Self } \\
\text { estimation }\end{array}$} & On a scale from 1 to 10 , how do you estimate your programming experience? & $\begin{array}{l}\text { 1: very inexperienced to } \\
10: \text { very experienced }\end{array}$ & s.PE \\
\hline & $\begin{array}{l}\text { How do you estimate your programming experience compared to experts with } \\
20 \text { years of practical experience? }\end{array}$ & $\begin{array}{l}\text { 1: very inexperienced to } \\
5: \text { very experienced }\end{array}$ & s.Experts \\
\hline & $\begin{array}{l}\text { How do you estimate your programming experience compared to your class } \\
\text { mates? }\end{array}$ & $\begin{array}{l}\text { 1: very inexperienced to } \\
\text { 5: very experienced }\end{array}$ & s.ClassMates \\
\hline & How experienced are you with the following languages: Java/C/Haskell/Prolog & $\begin{array}{l}\text { 1: very inexperienced to } \\
\text { 5: very experienced }\end{array}$ & $\begin{array}{l}\text { s.Java/s.C/s.Haskell/ } \\
\text { s.Prolog }\end{array}$ \\
\hline & $\begin{array}{l}\text { How many additional languages do you know (with medium experience or } \\
\text { better)? }\end{array}$ & Integer & s.NumLanguages \\
\hline & $\begin{array}{l}\text { How experienced are you with the following programming paradigms: } \\
\text { functional/imperative/logical/object-oriented programming? }\end{array}$ & $\begin{array}{l}\text { 1: very inexperienced to } \\
\text { 5: very experienced }\end{array}$ & $\begin{array}{l}\text { s.Functional/s.Imperative/ } \\
\text { s.Logical/s.ObjectOriented }\end{array}$ \\
\hline \multirow[t]{2}{*}{ Years } & For how many years have you been programming? & Integer & y.Prog \\
\hline & $\begin{array}{l}\text { For how many years have you been programming for larger software projects, } \\
\text { e.g., in a company? }\end{array}$ & Integer & y.ProgProf \\
\hline \multirow[t]{2}{*}{ Education } & What year did you enroll at university? & Integer & e.Years \\
\hline & How many courses did you take in which you had to implement source code? & Integer & e.Courses \\
\hline Size & How large were the professional projects typically? & $\begin{array}{l}\text { NA, }<900,900-40000 \\
>40000\end{array}$ & z.Size \\
\hline Other & How old are you? & Integer & o.Age \\
\hline
\end{tabular}

of the questionnaire we used in our experiment is available at the project's website. Next, we explain each question in detail.

\section{A. Years}

Questions of this category mostly referred to how many years a subject was programming in general and programming professionally. Programming in general includes the time when subjects started programming, which includes simple hello-world-like programs. Professional programming describes when subjects earned money for programming, which typically requires a certain experience level. In our questionnaire, we asked both questions. We believe that both questions are an indicator for programming experience, because the longer someone is programming, the more source code she has seen and implemented and, thus, the higher her programming experience should be.

\section{B. Education}

This category contains questions that assess educational aspects. We asked subjects to state the number of courses they took in which they had to implement source code and the year in which they enrolled. ${ }^{5}$ The number of courses roughly indicates how much source code subjects had implemented. With the years a subject is studying, we get an indicator of the education level: The longer a subject has been studying, the more experience she should have gained through her studies.

\section{Self Estimation}

In this category, we asked subjects to estimate their own experience level. With the first question, we asked subjects to estimate their programming experience on a scale from 1 to 10 .

\footnotetext{
${ }^{5}$ Recoded into number of years a subject has been enrolled.
}

We did not clarify what we mean by programming experience, but let subjects use their intuitive definition of programming experience. This way, we did not use a definition that felt unnatural for subjects. We used a 10-point scale to have a finegrained estimation. In the remaining questions, we used a fivepoint scale, because we think that a coarse-grained estimation is better for subjects to estimate their experience in these more specific questions.

Next, we asked subjects to set their programming experience in relation to experienced programmers and their class mates. This way, we aim at forcing subjects to think more thoroughly about their level of experience and set it into context.

Additionally, we asked subjects how familiar they are with certain programming languages. The more programming languages a developer is familiar with, the more she has learned about programming in general. Hence, her experience should be larger. Furthermore, the experience with the underlying programming language of the experiment can be assessed. Beyond that, we asked subjects to list the number of programming languages in which they are experienced at least to a mediocre level. This way, we can identify and assess familiarity with many languages without listing each existing programming language. The same applies to familiarity with different programming paradigms.

\section{Size}

We asked subjects with professional experience about the size of their projects. We used the categorization into small, medium, and large based on the lines of code according to van Mayrhauser and Vans [28].

In addition, we also included the age of subjects in the questionnaire. This way, we aim at having a more exhaustive understanding of programming experience. 


\section{EMPIRICAL VALIDATION}

Constructing and validating a questionnaire is a long and 3 tedious endeavor that requires several (replicated) experi- 4 ments [24]. In this paper, we start this process.

There are different ways to validate a questionnaire. For 6 example, we could recruit programming experts and novices as subjects and compare their answers in the questionnaire. Since 7 there is a considerable difference in the experience between 9 both groups, we should also see a similar difference in the 10 questionnaire. Another way is to compare the answers in the 11 questionnaire with performance in tasks that are related to 13 programming experience. The benefit is that we do not need 14 different groups of subjects; one group is sufficient. We used ${ }^{15}$ the latter way with a group of students, because we found in 16 our review that they are often used as subjects in software- 17 engineering experiments. Hence, they represent an important ${ }^{18}$ sample. Furthermore, students can be comparable to experts under certain conditions [18], [27].

Since we recruit students, we expect only little variation for some questions (e.g., o.Age, y.ProgProf). Nevertheless, we asked these questions anyway to have a more exhaustive data set. Of course, further experiments with different groups of subjects (e.g., professional programmers) are necessary. To this end, our experimental design can be reused, which we plan to do in future work.

To present our experiment, we use the guidelines suggested by Jedlitschka and Ciolkowski [19]. All material presented here (e.g., tasks, overview of statistical anaylsis) is available at the project's website.

\section{A. Objective}

With our experiment, we aim at evaluating how the questions relate to programming experience. To this end, we have to find an indicator of programming experience to which we can compare the answers of our programming-experience questionnaire. Hence, we designed a number of programming tasks that subjects should solve in a given time. For each task, we measure whether a subject solves a task correctly and how long she needs to complete a task. The first underlying assumption is that the more experienced subjects are, the more tasks they solve correctly. Since experienced subjects have seen more source code compared to inexperienced subjects, they should have less trouble in analyzing what source code does and, hence, solve more tasks correctly. The second assumption is that experienced subjects are faster in analyzing source code, because they have done it more often and know better what to look for.

As we are just starting the validation, we have no hypotheses about how our questions relate to the performance in the programming tasks.

\section{B. Material}

Overall, we designed 10 program-comprehension tasks. We gave subjects source code and asked to find out what the source code is doing or what would be printed. In Figure 2, we show the source code of the first task to give an impression

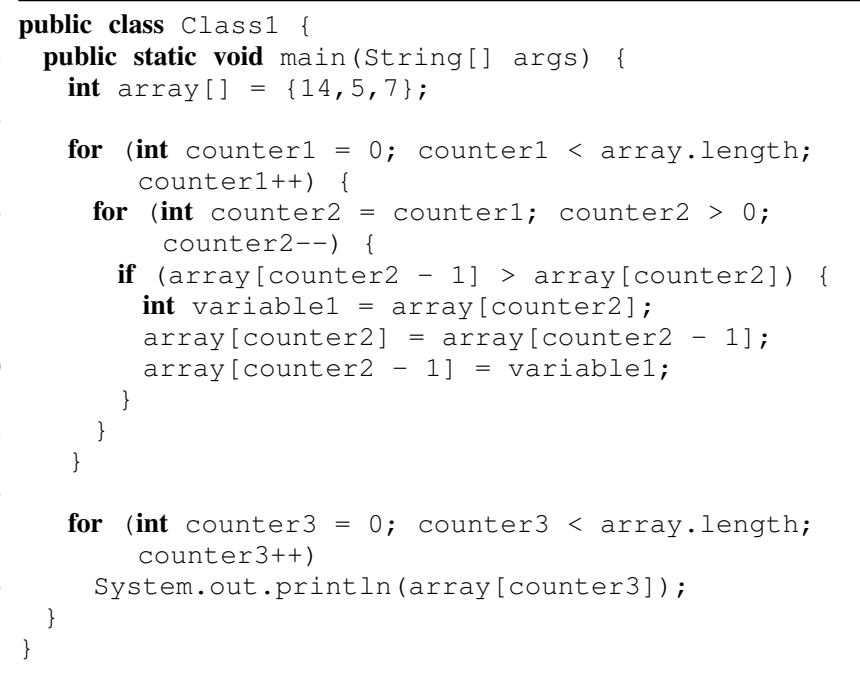

Fig. 2. Source code for first task.

of the nature of our tasks (all other tasks are available on the project's website). We asked subjects what executing this code would print. The source code sorts an array of numbers, so the correct answer is $5,7,14$. The remaining tasks were roughly similar: Two tasks were about a stack, five about a linked list, one involved command-line parameters, and the last was a bug fixing task. The tasks were presented in a fixed order; subjects could only go forward. To match the average experience level of undergraduates (who we recruited as subjects), we selected typical algorithms presented in introductory programming lectures. When replicating this experiment with programming experts, it might be necessary to adjust the tasks to better match the high level of experience. Only the last two tasks required a higher experience level. In Task 9, we used command-line parameters, which are not typically taught at sophomore level. In the last task, we use source code of MobileMedia, a software for manipulating multi-media data on mobile devices [14]. It consists of 2,800 lines of code in 21 classes. We included the last two tasks to identify highly experienced subjects among sophomores, since some students start to program before they begin to study. Only highly experienced subjects should be able to complete this task. All source code was in Java, the language that subjects were most familiar with.

We designed the tasks such that only experienced subjects would be able to complete all tasks, which we confirmed in a pre test with PhD students from the University of Magdeburg. This way, we can better differentiate between high and low experienced subjects. To make sure that subjects are not disappointed with their performance in the experiment, we explained that they would not be able to solve all tasks, but should simply proceed as far as possible within given time.

To present the questionnaire, tasks, and source code to subjects, we used our own tool infrastructure PROPHET [12]. It displays source code and tasks, lets subjects enter answers, and $\operatorname{logs}$ the time subjects spend on each task, in addition to 
the behavior of subjects, such as opening files. This way, we control the influence of subjects' familiarity with an IDE. ${ }^{6}$

\section{Subjects}

Subjects came from the University of Passau (27), Philipps University Marburg (31), and University of Magdeburg (70), so we had 128 subjects in total. All universities are located in Germany. Subjects from Passau and Marburg were in the end of their third semester and attended a course on software engineering. Subjects from Magdeburg were at the beginning of their fourth semester and from different courses. The level of education of all subjects was comparable, because no courses took place between semesters and subject had to complete similar courses at all universities. All subjects were offered different kinds of bonus points for their course (e.g., omitting one homework assignment) for participating in the experiment independent of their performance. All subjects participated voluntarily, were aware that they took part in an experiment, and could quit anytime. Data was logged anonymously and matched with a subject code.

Since, we recruited subjects from different universities, we actually have different samples. However, only the question s.ClassMates is specific for each university, because subjects can only compare themselves to the students of their university. To test whether our samples differ in that question, we conducted a Kruskal-Wallis test for s.ClassMates, which revealed no significant differences between the three universities $\left(\chi^{2}=\right.$ 1.275, $\mathrm{df}=2, \mathrm{p}=0.529$ ) [1]. Hence, we can treat our three samples as one sample.

\section{Execution}

The experiments took place in January and April 2011 at the Universities of Passau, Marburg, and Magdeburg as part of a regular lecture or exercise session. It started with letting the subjects complete the programming-experience questionnaire without knowing its specific purpose. Then, we gave subjects an introduction about the general purpose and proceeding of the experiment, without mentioning our goal. The introduction was given by the same experimenter each time. After all questions were answered, subjects started to work on the tasks on their own. Since we had time constraints, the time limit for the experiment was set to 40 minutes. After time ran out, subjects were allowed to finish the task they were currently working on. Two to three experimenters checked that subjects worked as planned.

\section{E. Deviation}

We had a technical error for the presentation of the programming-experience questionnaire, such that we could not measure s.PE for all subjects. As soon as we noticed that, we fixed the problem. However, we only have the answer of 70 out of 128 subjects for this question.

\footnotetext{
${ }^{6}$ There may be other confounding parameters; our sample is large enough to control their influence.
}

\begin{tabular}{|c|c|c|c|c|}
\hline Variable & $\begin{array}{l}\text { Response time } \\
\text { Distribution }\end{array}$ & Mean & $\mathrm{N}$ & Correct \\
\hline Task 1 & $\vdash-\square-1 \infty$ & 4.44 & 124 & 70 \\
\hline Task 2 & $+\mathbb{T}-+\infty$ & 3.65 & 123 & 90 \\
\hline Task 3 & 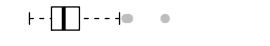 & 5.02 & 121 & 97 \\
\hline Task 4 & $r-\square \square \cdots+$ & 6.17 & 117 & 22 \\
\hline Task 5 & $+-\square-\cdots$ & 4.06 & 118 & 46 \\
\hline Task 6 & $+-\square \square-\cdots+-$ & 4.72 & 111 & 40 \\
\hline Task 7 & $r \mathbb{W}-\div \quad \circ \quad \circ$ & 2.34 & 92 & 31 \\
\hline Task 8 & 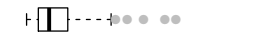 & 4.1 & 82 & 69 \\
\hline Task 9 & $+\mathbb{I}-+\infty$ & 1.94 & 78 & 11 \\
\hline \multirow[t]{2}{*}{ Task 10} & 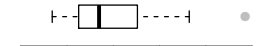 & 9.64 & 30 & 22 \\
\hline & \begin{tabular}{l|l|l|l|l|} 
& 1 & & 1 & \\
\end{tabular} & & & \\
\hline
\end{tabular}

TABLE II

OVERVIEW OF RESPONSE TIME FOR EACH TASK.

\section{DesCRIPTIVE Statistics}

First, we describe descriptive statistics to get an overview of our data. Second, we present how each question correlates with the performance in the tasks. This way, we get an impression of how important each question is as indicator for programming in our sample.

\section{A. Means and Standard Deviations}

In Table II, we give an overview of how subjects solved the tasks. Column Mean contains the average time in minutes of subjects who completed a task. Since not all subjects finished all tasks, they cannot be interpreted across tasks. We discuss the most important values. Task 10 took the longest time to complete (on average, 9.6 minutes). This is caused by the large underlying size of the source code for the last task with over 2000 lines of code. To solve Task 9, subjects needed on average 1.9 minutes; most likely, because its source code consisted of only 10 lines. Furthermore, only 11 subjects solved it correctly. To solve this task, subjects must be familiar with command-line parameters, which is not typical for the average sophomore. Considering the correctness of Task 4, we see that only 22 subjects solved this task correctly. In this task, elements were added to an initially empty linked list, such that the list is sorted in a descending order after the insertion. In most of the wrong answers, we found that the order of the elements was wrong. We believe that subjects did not analyze the insert algorithm thoroughly enough and assumed an ascending order of elements.

In Fig. 3, we show the number of correctly solved tasks per subject. As we expected, none of our subjects solved 9 or 10 tasks correctly. Especially the last two tasks (task 9: commandline parameters; task 10: 2,800 lines of code) required an experience level beyond that of undergraduate students. More than half of the students (72) solved two to four tasks correctly. Taking into account the time constraints (40 minutes to solve 


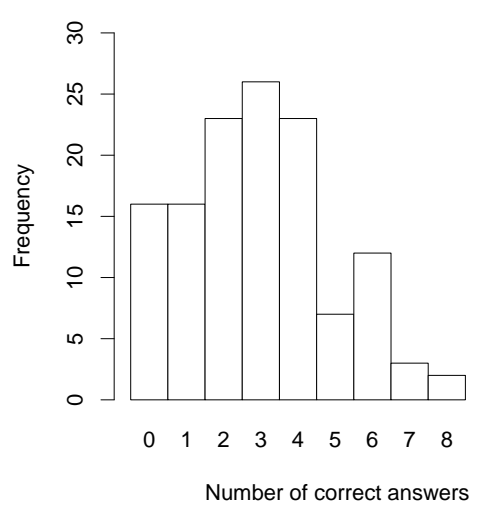

Fig. 3. Frequencies of number of correct answers.

\begin{tabular}{|c|c|c|c|c|}
\hline No. & Question & Distribution & & $\mathrm{N}$ \\
\hline 1 & s.PE & $\square \square \square \square \square$ & & 70 \\
\hline 2 & s.Experts & & & 126 \\
\hline 3 & s.ClassMates & 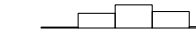 & & 127 \\
\hline 4 & s.Java & $\Longrightarrow$ & & 124 \\
\hline 5 & s.C & $\square \square$ & & 127 \\
\hline 6 & s.Haskell & $\square \longdiv { \square }$ & & 128 \\
\hline 7 & s.Prolog & $\square$ & & 128 \\
\hline 8 & s.NumLanguages & $0 \square \square$ & 6 & 118 \\
\hline 9 & s.Functional & $\square \square$ & & 127 \\
\hline 10 & s.Imperative & $\square \longdiv { \square }$ & כ & 128 \\
\hline 11 & s.Logical & ما & & 126 \\
\hline 12 & s.ObjectOriented & 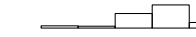 & & 127 \\
\hline 13 & y.Prog & $0+\square-\cdots+\cdots$ & 25 & 123 \\
\hline 14 & y.ProgProf & $0 D_{-\dashv} \cdots \cdots$ & 7 & 127 \\
\hline 15 & e.Years & $0 \mid \square-+。$ & 9 & 126 \\
\hline 16 & e.Courses & $0 \mid 1-\mathbb{T}^{---100}$ & 20 & 123 \\
\hline 17 & o.Size & \begin{tabular}{|r|r} 
\\
\end{tabular} & & 128 \\
\hline 18 & o.Age & $19+-\square--+\cdots \cdots$ & 40 & 128 \\
\hline
\end{tabular}

TABLE III

OVERVIEW OF ANSWERS IN QUESTIONNAIRE.

10 tasks), it is not surprising that the number of tasks that a student solved correctly is rather low.

In Table III, we show the answers subjects gave in our questionnaire. The median for the first self estimation varies between 2 and 3, which we would expect from sophomores. In general, subjects felt very inexperienced with logical programming and experienced with object-oriented programming. The median of how long subjects are programming is 4 , but only few subjects said they were programming for more then 10 years. Although subjects took a sophomore course, some subjects were enrolled for more than 3 years, ${ }^{7}$ which could also explain why some subjects completed numerous courses in which they had to implement source code.

\footnotetext{
${ }^{7}$ The German system allows subjects to take courses in somewhat flexible order and timing.
}

\begin{tabular}{rlrr}
\hline No. & Question & $\rho$ & $\mathrm{N}$ \\
\hline 1 & s.PE & .539 & 70 \\
2 & s.Experts & .292 & 126 \\
3 & s.ClassMates & .403 & 127 \\
4 & s.Java & .277 & 124 \\
5 & s.C & .057 & 127 \\
6 & s.Hasekll & .252 & 128 \\
7 & s.Prolog & .186 & 128 \\
8 & s.NumLanguages & .182 & 118 \\
9 & s.Functional & .238 & 127 \\
10 & s.Imperative & .244 & 128 \\
11 & s.Logical & .128 & 126 \\
12 & s.ObjectOriented & .354 & 127 \\
13 & y.Prog & .359 & 123 \\
14 & y.ProgProf & .004 & 127 \\
15 & e.Years & -.058 & 126 \\
16 & e.Courses & .135 & 123 \\
17 & z.Size & -.108 & 128 \\
18 & o.Age & -.116 & 128 \\
\hline$\rho:$ Spearman correlation; & N: number of subjects; \\
gray cells denote significant correlations $(p<.05)$.
\end{tabular}

TABLE IV

SPEARMAN CORRELATIONS OF NUMBER OF CORRECT ANSWERS WITH ANSWERS IN QUESTIONNAIRE.

\section{B. Correlations}

In Table IV, we give an overview of the correlation of the number of correct answers with the answers of the questionnaire. Since we correlate ordinal data, we use the Spearman rank correlation [1]. For about half of the questions of self estimation, we obtain small to strong correlations. ${ }^{8}$ The highest correlation with number of correct answer has s.PE. The lowest significant correlation is with s.NumLanguages. Regarding y.Prog and y.ProgProf, we have medium correlations with the number of correct answers. E. Years does not correlate with the number of correct answers. For the remaining questions, we do not observe significant correlations.

For completeness, we show the correlations of response time with each of the questions of our questionnaire in Table V. Only 23 correlations, of 180, are significant, which is in the range of coincidence, given the typical $\alpha$ level of 0.05 . Since there are so many correlations, a meaningful interpretation is impossible without further analysis, for example a factor analysis. However, such analysis typically requires a large number of subjects. Since we have a decreasing number of subjects with each task, we leave analyzing the response times for future experiments.

\section{EXPLORATORY ANALYSIS}

In this section, we explore the data. For this analysis, we excluded question s.PE, because the methods we use require a large number of subjects, but we only had 70 subjects who answered this question (cf. Section IV-E). Furthermore, we only use the number of correct answers as indicator for program comprehension, but not time, since only few subjects completed all tasks. We decided not to compute the average response time for a task or to analyze the response times for

\footnotetext{
${ }^{8}$ Small: \pm 0.1 to \pm 0.3 ; medium: \pm 0.3 to \pm 0.5 ; strong: \pm 0.5 to $\pm 1[8]$.
} 


\begin{tabular}{|c|c|c|c|c|c|c|c|c|c|c|c|c|}
\hline No. & Question & Task 1 & Task 2 & Task 3 & Task 4 & Task 5 & Task 6 & Task 7 & Task 8 & Task 9 & Task 10 & Number of subjects \\
\hline 1 & s.PE & -.279 & -.417 & -.042 & .004 & -.002 & .016 & .014 & -.182 & .071 & .085 & $68-27$ \\
\hline 2 & s.Experts & -.300 & -.177 & .047 & -.026 & .006 & -.075 & -.217 & -.004 & .206 & .131 & $122-40$ \\
\hline 3 & s.ClassMates & -.189 & -.401 & -.084 & -.065 & -.053 & -.059 & -.163 & -.061 & .161 & .100 & $123-40$ \\
\hline 4 & s.Java & .029 & -.066 & -.154 & -.022 & -.066 & .003 & -.040 & .145 & -.170 & -.222 & $105-34$ \\
\hline 5 & s.C & -.175 & -.124 & .018 & .027 & .126 & -.108 & -.056 & -.052 & .043 & .108 & $123-40$ \\
\hline 6 & s.Haskell & -.171 & -.109 & -.144 & -.113 & -.014 & -.216 & -.153 & -.183 & .019 & .158 & $124-40$ \\
\hline 7 & s.Prolog & -.174 & -.141 & -.079 & -.104 & -.027 & -.039 & .076 & -.239 & -.047 & .146 & $124-40$ \\
\hline 9 & s.Functional & -.148 & -.150 & -.150 & -.004 & -.017 & -.204 & -.120 & -.217 & .027 & .175 & $123-40$ \\
\hline 10 & s.Imperative & -.283 & .331 & -.033 & -.089 & -.06 & -.129 & -.296 & -.156 & .126 & .043 & $124-40$ \\
\hline 11 & s.Logical & -.209 & -.105 & -.158 & -.136 & -.022 & -.014 & .058 & -.257 & -.191 & .108 & $122-40$ \\
\hline 12 & s.ObjectOriented & -.084 & -.232 & -.008 & .012 & -.093 & -.034 & .025 & -.060 & .156 & .082 & $123-40$ \\
\hline 13 & y.Prog & -.241 & -.379 & -.144 & -.071 & .010 & -.113 & -.258 & -.159 & .273 & .180 & $120-38$ \\
\hline 14 & y.ProgProf & -.217 & -.196 & -.012 & -.119 & -.130 & .071 & -.274 & -.022 & .044 & -.010 & $123-39$ \\
\hline 15 & e.Years & -.032 & .001 & .018 & -.152 & .059 & .047 & -.119 & .037 & -.092 & -.173 & $122-40$ \\
\hline 17 & z.Size & -.155 & -.160 & -.057 & -.134 & .059 & .003 & -.201 & .046 & .000 & -.023 & $124-40$ \\
\hline 18 & o.Age & .036 & .014 & .110 & .082 & .131 & .102 & -.081 & .090 & .059 & .010 & $124-40$ \\
\hline
\end{tabular}

TABLE V

SPEARMAN CORRELATIONS OF RESPONSE TIMES FOR EACH TASK WITH ANSWERS IN QUESTIONNAIRE.

each task, because that would be too inaccurate. Furthermore, we would have to compute efficiency measure to deal with wrong answers [29], but it is not clear what they describe.

For exploration, we use stepwise regression and exploratory factor analysis. Both approaches are standard in psychology, but rarely used in software-engineering research. Therefore, we start each section with an overview of the methods, before we present and interpret the results.

\section{A. Stepwise Regression}

\section{Overview}

So, which questions are the best indicators for programming experience? The first obvious selection criterion is to include all questions that have a high correlation with the number of correctly solved task. However, the questions themselves might correlate with each other. For example, the s.ClassMates correlates with s.ObjectOriented with 0.552 . Hence, we can assume both questions are not independent from each other. If we used both questions as indicator, we would overestimate the relationship of both questions with programming experience, that is we would count the common part of both questions twice, although we should count it only once.

To account for the correlations between questions, we use stepwise regression [22]. Stepwise regression builds a model of the influence of the questions on the number of correct answers in a stepwise manner. It starts by including the question with the highest correlation, which, in our case, is s.ClassMates. Then, it considers the question with the next highest correlation, which is y.Prof. Using this question, it computes the partial correlation with the number of correct answers, describing the correlation of two variables cleaned from the influence of a third variable [9]. Thus, the correlation of y.Prog with the number of correct answers, cleaned from the influence of s.ClassMates, is computed. If this cleaned

\begin{tabular}{llll}
\hline Question & Beta & $\mathrm{t}$ & $\mathrm{p}$ \\
\hline s.ClassMates & .441 & 3.219 & .002 \\
s.Logical & .286 & 2.241 & .030 \\
\hline \multicolumn{5}{c}{ TABLE VI }
\end{tabular}

RESULTING MODEL OF STEPWISE REGRESSION.

correlation is high enough, the question is included, else it is excluded. The goal is to include questions with a high correlation with the number of correct answers, such that as few questions as possible are selected to have a model as parsimonious as possible. This is repeated with all questions of the questionnaire.

\section{Results and Interpretation}

In Table VI, we show the results for our questionnaire. With stepwise regression (specifically, we used stepwise as inclusion method), we extracted two questions: Experience with logical programming (s.Logical) and the self-estimated experience compared to class mates (s.ClassMates). The higher the Beta value, the larger is the influence of a question on the number of correctly solved tasks. The model is significant $\left(F_{2,45}=8.472, p<.002\right)$ and the adjusted $R^{2}$ is 0.241 , meaning that we explain $24.1 \%$ of the variance in the number of correct answers with our model (explaining the meaning of the values exceeds the scope of this paper; see [22]).

Hence, the result of the stepwise-regression algorithm is that questions s.ClassMates and s.Logical contribute most to the number of correct answers: The higher subjects estimate their experience compared to class mates and their experience with logical programming, the more tasks they solve correctly. We believe that stepwise regression extracted s.ClassMates, and not s.PE or s.Experts, because we recruited students as subjects and the tasks are taken from introductory programming 
lectures. Hence, if a subject estimates her experience better than her class mates, she should be better in solving the tasks. However, we need further research to be sure about that.

Why was s.Logical extracted and not s.Java, which is closer to our experiment? We believe that the reason is that our subjects learn Java as one of their first programming language and feel somewhat confident with it. In contrast, learning a logical programming language is only a minor part of the curriculum of all three universities. Hence, if students estimate that they are familiar with logical programming, they may have an interest in learning other ways of programming and pursue it, which increases their programming experience.

The model received from stepwise regression describes Beta values, which are weights for each question. For example, if a subject estimates a 4 in s.ClassMates (more experienced than class mates) and a 2 in s.Logical (unfamiliar with logical programming), the resulting value for programming experience is $0.441 * 4+0.286 * 2=2.336$ (we omitted a constant to add as part of the mode for simplicity).

Hence, we have identified two questions that explain $24.1 \%$ of the variance of the number of correct answers. We could include more questions to improve the amount of explained variance, but none of the questions contribute a significant amount of variance. Since a model should be parsimonious, stepwise regression excluded all other questions. Thus, for our sample, these two questions provide the best indicators for programming experience.

\section{B. Exploratory Factor Analysis}

\section{Overview}

Furthermore, to look for a pattern in our questions, we analyzed whether questions in our questionnaire correlate. To this end, we conducted an exploratory factor analysis [2]. The goal is to reduce a number of observed variables to a small number of underlying latent variables or factors (i.e., variables that cannot be observed directly). To this end, the correlations of the observed variables are analyzed to identify groups of variables that correlate among each other. For example, experience with Haskell and functional programming are very similar and might be explained by a common underlying factor. The result of an exploratory factor analysis is a number of factors that summarize observed variables into groups. However, the meaning of the factors is not a result of the analysis, but relies on interpretation.

\section{Results and Interpretation}

In Table VII, we show the results of our exploratory factor analysis. The numbers in the table denote correlations or factor loadings of the variables in our questionnaire with identified factors. By convention, factor loadings that have an absolute of smaller than .32 are omitted, because they are too small to be relevant [10]. There are main loadings, which are the highest factor loading of one variable, and cross loadings, which are all other factor loadings of a variable that have an absolute of more then .32. The higher the main loading and the smaller the number of cross loadings, the more unambiguously the

\begin{tabular}{|c|c|c|c|c|c|}
\hline Variable & Factor 1 & Factor 2 & Factor 3 & Factor 4 & Factor 5 \\
\hline s.C & .723 & & & & \\
\hline s.ObjectOriented & .700 & & & .403 & \\
\hline s.Imperative & .673 & .333 & & .303 & \\
\hline s.Experts & .600 & .326 & & & \\
\hline s.Java & .540 & & .427 & & \\
\hline y.ProgProf & & .859 & & & \\
\hline z.Size & & .764 & & & \\
\hline s.NumLanguages & .335 & .489 & & .403 & \\
\hline s.ClassMates & & .449 & .403 & .424 & \\
\hline s.Functional & & & .880 & & \\
\hline s.Haskell & & & .879 & & \\
\hline e.Courses & & & & .795 & \\
\hline e.Years & & & -.460 & .573 & \\
\hline y.Prog & & .493 & & .554 & \\
\hline s.Logical & & & & & .905 \\
\hline s.Prolog & & & & & .883 \\
\hline
\end{tabular}

TABLE VII

FACTOR LOADINGS OF VARIABLES IN QUESTIONNAIRE.

influence of one factor on a variable is. If a variable has many cross loadings, it is unclear what it exactly measures and more investigations on this variable are necessary in subsequent experiments.

The first factor of our analysis summarizes the variables s.C, s.ObjectOriented, s.Imperative, s.Experts, and s.Java. This means that these variables have a high correlation amongst each other and can be described by this factor. Except for s.Experts, this seems to make sense, because $\mathrm{C}$ and Java and the corresponding paradigms are similar and often taught at universities. We conjecture that s.Experts also loads on this factor, because it explains the confidence level with mainstream programming languages. We can name this factor experience with mainstream languages.

The second factor contains the variables y.ProgProf, z.Size, s.NumLanguages, and s.ClassMates. These variables fit together well, because the longer a subject is programming professionally, the more likely she has worked with large projects and the more language she has encountered. Additionally, since it is not typical for undergraduates to program professionally, subjects that have programmed professionally estimate their experience higher compared to their class mates. We can name this factor professional experience.

Factor three and five group s.Functional/s.Haskell and s.Logical/s.Prolog in an intuitive way. Hence, we name these factors functional experience and logical experience.

The fourth factor summarizes the variables e.Courses, e.Years, and y.Prog, which are all related to the subject's education. We can name this factor experience from education.

Now, we have to take a look at the cross loadings. As an example, we look at e.Years, which also loads on functional experience. This means that part of this variable can also be explained by this factor. Unfortunately, we cannot unambiguously define to which factor this variable belongs best, we can only state e. Years has a higher loading of factor experience 
from education. This could also mean that we need two factors to explain this variable. However, with a factor analysis, we are looking for a parsimonious model without having more relationships than necessary.

To summarize the exploratory factor analysis, we extracted five factors: experience with mainstream languages, professional experience., functional experience, experience from education, and logical experience that summarize the questions of our questionnaire in our sample.

\section{Threats to VALIDity}

In this section, we discuss threats to validity, which is common for every experiment. First, the selection of tasks threatens internal validity. With other tasks, results may look different. However, we made sure to select tasks representative for the experience level of undergraduates and with varying difficulty. Thus, more experienced subjects should perform better than less experienced subjects. Hence, our task selection is appropriate for our purpose.

The major threat to external validity is our sample selection: We only recruited undergraduate students Our results can be interpreted only in the context of subjects with similar experience. When applying the results to professional programmers, other indicators, such as the years of programming (professionally) may be a better indicator than self estimation. However, since most experiments are conducted with students, our results are useful for many researchers.

Furthermore, the results of our exploratory analysis cannot be generalized without confirmatory analysis based on further experiments. If we used the same data set, we could not show that our model is valid in general, but for our specific data set. However, confirmatory analysis requires considerable effort, because we have to conduct another experiment. Furthermore, we need a large sample, depending on the complexity of the model. In our case, we would need at least 80 subjects to confirm our model (i.e., the five factors with the different questions that load on them) [4].

\section{RECOMMENDATIONS}

So far, we have combined different questions from different categories found in literature into a single questionnaire. We conducted a controlled experiment with undergraduate students and explored our data for initial validation. What have we learned in terms of recommendations for future research?

First, we showed that in literature, there are many different ways to measure and control programming experience. Furthermore, in many cases, the methods are not reported. We recommend to mix questions from different categories into a single questionnaire, of which we presented a draft. We recommend to report precisely which measure was used and how groups have been formed according to it. This helps to judge validity and compare and interpret multiple studies.

Second, we can recommend self-estimation questions to judge programming experience among undergraduate students. In our experiment, several self-estimation questions correlated to a strong to medium degree (s.PE: .539; s.ClassMates: .403;
s.ObjectOriented: .354) with the number of correct answers much more than questions regarding the categories education, size, and other. Among undergraduate students, answers to questions from the latter categories differ only slightly. The only medium correlation beyond self estimation is y.Prog (.359), the number of years a subject is programming at all.

Third, if resource constraints allow it, researchers can combine multiple questions, of which some serve as control questions to see whether subjects answered honestly, which is custom in designing questionnaires [24]. For example, in our case, when using s.PE, s.ClassMates and s.ObjectOriented are suitable as control questions, since they both show a strong correlation with s.PE (s.ClassMates: .625; s.ObjectOriented: .696).

Fourth, since correlations between questions confound the strength of a question as indicator for programming experience (cf. Section VI-A), we extracted two relevant questions, s.ClassMates and s.Logical, that together serve as best indicator to predict the number of correct answers in our experiment (each question can be supplied with control questions if resource constraints allow it).

Fifth, our exploratory analysis indicates five factors for programming experience that can serve as starting point for developing a theory on programming experience. The results do not help building a survey right away, but with additional confirmation, e.g., confirmatory factor analysis on another data set, they can help understanding how programming experience works and which kinds of questions query relevant parameters. However, to that end, there is still a long way.

Overall note that while our literature review and the construction of the questionnaire are intended for measuring programming experience in general, we only validated it for a specific setting: predicting programming experience among a homogeneous group of undergraduate students. Hence, our recommendations remain limited to this setting. We conjecture that with experienced programmers, questions from the categories education, years, and size have more predictive power. Whether self-estimation remains a good indicator in this setting remains an open question for future work.

We plan to validate the questionnaire with other groups in further experiments. In this validation, we will reuse the experimental design and methodology developed in this work.

\section{RELATED WORK}

In general, related work to ours evaluated possible criteria that can be used to categorize subjects upfront. For example, Kleinschmager and Hanenberg analyzed the influence of self-estimation, university grades, and pre-tests on historical data for programming experiments [21]. To this end, they analyzed the data of two previously conducted programming experiments with students as subjects. They compared selfestimation, university grades, and pretests with the performance of subjects in the experiments and found that selfestimation was not worse than university grades or pre-tests in order to categorize subjects. These results complement ours, as we did not look into pretests and grades. 
Höst et al. analyze the suitability of students as subjects [18] by comparing the performance of students and professional software developers for non-trivial tasks regarding judgment about factors affecting the lead-time of software-development projects. They found no differences between groups. Thus, classification of subjects had no effect on their performance.

Bornat et al. used a pre-test to categorize god and bad novice programmers [6]. It relates to our work, in that we also aim at measuring good and bad programmers, with the difference that we seek a simple-to-apply questionnaire.

\section{$\mathrm{X}$. Conclusion}

The understanding of programming experience is diverse. Often, researchers do not specify their understanding of programming experience or do not consider it at all. However, this threatens the validity of experiments and makes interpretations across different experiments difficult.

In a controlled experiment, we evaluated the measurement of programming experience found in literature. We found that within groups of undergraduate students, self estimation indicates programming experience well. Specifically, we extracted programming experience compared to class mates and experience with logical programming as relevant questions. In conjunction with control questions, such programming experience in general or experience with Prolog, they can be used as indicator for programming experience. Furthermore, we extracted a five-factor model with the factors experience with mainstream languages, professional experience, functional experience, experience from education, logical experience. The next steps are confirmatory analyses, in which we aim at confirming the results of our experiment with different groups of subjects. To this end, we and other research groups can reuse our experimental design.

\section{ACKNOWLEDGMENTS}

Feigenspan's work is supported by BMBF project 01IM10002B, Kästner's work partly by ERC (\#203099), and Apel's work by the DFG projects \#AP 206/2 and \#AP 206/4. Thanks to Jana Schumann for her support in the literature study. We thank all experimenters for their support in setting up and conducting the experiment.

\section{REFERENCES}

[1] T. Anderson and J. Finn. The New Statistical Analysis of Data. Springer, 1996.

[2] T. W. Anderson and H. Rubin. Statistical Inference in Factor Analysis. In Proc. Berkeley Symposium on Mathematical Statistics and Probability, Volume 5, pages 111-150. University of California Press, 1956.

[3] E. Arisholm. Evaluating Pair Programming with Respect to System Complexity and Programmer Expertise. IEEE Trans. Softw. Eng., 33(2):65-86, 2007.

[4] P. Bentler. Practical Issues in Structural Modeling. Sociological Methods Research, 16(1):78-117, 1987.

[5] S. Biffl and W. Grossmann. Evaluating the Accuracy of Defect Estimation Models Based on Inspection Data from Two Inspection Cycles. In Proc. Int'l Conf. Software Engineering (ICSE), pages 145154. IEEE CS, 2001.

[6] R. Bornat, S. Dehnadi, and Simon. Mental Models, Consistency and Programming Aptitude. In Proc. Conf. on Australasian Computing Education - Volume 78, pages 53-61. Australian Computer Society, Inc., 2008.
[7] C. Bunse. Using Patterns for the Refinement and Translation of UML Models: A Controlled Experiment. Empirical Softw. Eng., 11(2):227267,2006

[8] J. Cohen. Statistical Power Analysis for the Behavioral Sciences. Routledge Academic Press, second edition, 1988.

[9] J. Cohen and P. Cohen. Applied Multiple Regression: Correlation Analysis for the Behavioral Sciences. Addison Wesley, second edition, 1983.

[10] A. B. Costello and J. W. Osborne. Best Practices in Exploratory Factor Analysis: Four Recommendations for Getting the Most from your Analysis. Practical Assessment, Research \& Evaluation, 10(7):173-178, 2005.

[11] H. Erdogmus, M. Morisio, and M. Torchiano. On the effectiveness of the test-first approach to programming. IEEE Trans. Softw. Eng., 31(3):226-237, 2005.

[12] J. Feigenspan et al. PROPHET: Tool Infrastructure To Support Program Comprehension Experiments. Int'l Symposium Empirical Software Engineering and Measurement (ESEM), Poster.

[13] J. Feigenspan et al. Using Background Colors to Support Program Comprehension in Software Product Lines. In Proc. Int'l Conf. Evaluation and Assessment in Software Engineering (EASE), pages 66-75. Institution of Engineering and Technology, 2011.

[14] E. Figueiredo et al. Evolving Software Product Lines with Aspects: An Empirical Study on Design Stability. In Proc. Int'l Conf. Software Engineering (ICSE), pages 261-270. ACM Press, 2008.

[15] C. Goodwin. Research in Psychology: Methods and Design. Wiley Publishing, Inc., second edition, 1999.

[16] S. Hanenberg, S. Kleinschmager, and M. Josupeit-Walter. Does AspectOriented Programming Increase the Development Speed for Crosscutting Code? An Empirical Study. In Proc. Int'l Symposium Empirical Software Engineering and Measurement (ESEM), pages 156-167. IEEE CS, 2009.

[17] J. Hannay et al. Effects of Personality on Pair Programming. IEEE Trans. Softw. Eng., 36(1):61-80, 2010.

[18] M. Höst, B. Regnell, and C. Wohlin. Using Students as Subjects - A Comparative Study of Students and Professionals in Lead-Time Impact Assessment. Empirical Softw. Eng., 5(3):201-214, 2000.

[19] A. Jedlitschka, M. Ciolkowski, and D. Pfahl. Reporting Experiments in Software Engineering. In Guide to Advanced Empirical Software Engineering, pages 201-228. Springer, 2008.

[20] B. Kitchenham and S. Charters. Guidelines for Performing Systematic Literature Reviews in Software Engineering. Technical Report EBSE 2007-001, 2007.

[21] S. Kleinschmager and S. Hanenberg. How to Rate Programming Skills in Programming Experiments? A Preliminary, Exploratory, Study Based on University Marks, Pretests, and Self-Estimation. In Proc. ACM SIGPLAN Workshop on Evaluation and Usability of Programming Languages and Tools, pages 15-24. ACM Press, 2011.

[22] M. Lewis-Beck. Applied Regression: An Introduction. Sage Pubications, 1980.

[23] M. Müller. Are Reviews an Alternative to Pair Programming? Empirical Softw. Eng., 9(4):335-351, 2004.

[24] R. Peterson. Constructing Effective Questionnaires. Sage Publications, 2000.

[25] F. Ricca et al. The Role of Experience and Ability in Comprehension Tasks Supported by UML Stereotypes. In Proceedings of the 29th international conference on Software Engineering, pages 375-384. IEEE CS, 2007.

[26] J. Sillito, G. C. Murphy, and K. De Volder. Asking and Answering Questions during a Programming Change Task. IEEE Trans. Softw. Eng., 34(4):434-451, 2008.

[27] W. Tichy. Hints for Reviewing Empirical Work in Software Engineering. Empirical Softw. Eng., 5(4):309-312, 2000.

[28] A. von Mayrhauser and M. Vans. Program Comprehension During Software Maintenance and Evolution. Computer, 28(8):44-55, 1995.

[29] J. Yellott. Correction for Fast Guessing and the Speed Accuracy Tradeoff in Choice Reaction Time. Journal of Mathematical Psychology, 8:159-199, 1971 